\title{
Problemas relacionados con el consumo de alcohol en jóvenes de la provincia de Jujuy, Argentina
}

\author{
Ethel Alderete, MPH, PhD, ${ }^{(1)}$ Celia P Kaplan, PhD, MA, (2) Gregory Nah, MA, ${ }^{(2)}$ Eliseo J Pérez-Stable, MD. ${ }^{(2)}$
}

\author{
Alderete E, Kaplan CP, Nah G, Pérez-Stable EJ. \\ Problemas relacionados con el consumo de alcohol \\ en jóvenes de la provincia de Jujuy, Argentina. \\ Salud Publica Mex 2008;50:300-307.
}

\section{Resumen}

Objetivo. Examinar los patrones de consumo y los problemas relacionados con el alcohol en jóvenes de la provincia de Jujuy,Argentina. Material y métodos. En el año 2005 se aplicó una encuesta a una muestra representativa de 2924 jóvenes de noveno grado (I2-I7 años), incluidas la información sociodemográfica y de consumo y la prueba AUDIT-C. Resultados. Hasta $9 \%$ de las mujeres y $11 \%$ de los varones refirieron consumo de riesgo; asimismo, $12 \%$ de las mujeres y $19 \%$ de los varones notificaron síntomas de dependencia. Las mujeres presentaban menor probabilidad que los varones de experimentar síntomas de dependencia (RM ajustada $0.7 ;$ IC95\% 0.6-0.8) o de consumo perjudicial (RM ajustada $0.7 ;$ IC95\% 0.6-0.8). Tener mayor edad, trabajar y asistir al turno nocturno fueron factores de riesgo para el consumo de riesgo, síntomas de dependencia y consumo perjudicial. Conclusiones. Los resultados ponen de relieve la importancia de instituir intervenciones de prevención y tratamiento para los adolescentes.

Palabras clave: alcoholismo; adolescentes; América Latina; Argentina
Alderete E, Kaplan CP, Nah G, Pérez-Stable EJ. Problems related to alcohol drinking among youth in Jujuy, Argentina.

Salud Publica Mex 2008;50:300-307.

\section{Abstract}

Objective. To examine drinking patterns and alcohol-related problems among youth in Jujuy, Argentina. Material and Methods. A survey was conducted in 2005 with a representative sample of $9^{\text {th }}$ grade youth (12 to 17 years old) including sociodemographic and consumption data, and the AUDIT-C test. Results. Nine percent of girls and II\% of boys reported hazardous drinking; I $2 \%$ of girls and 19\% of boys reported dependence symptoms. The odds ratio for dependence symptoms (adjusted OR $0.7 ; 95 \% \mathrm{Cl}: 0.6-0.8$ ) and for hazardous drinking (adjusted OR $0.7 ; 95 \% \mathrm{Cl}: 0.6-0.8$ ) was significantly lower for girls compared with boys. Older age, working, and attending night school were risk factors for hazardous drinking, dependence symptoms, and harmful drinking. Conclusions. A significant proportion of youth reported problematic patterns of alcohol drinking, highlighting the need to implement prevention and treatment interventions tailored to the adolescent population.

Key words: alcoholism; adolescent; Latin America;Argentina

(I) Consejo Nacional de Investigaciones Científicas y Técnicas (CONICET). Argentina.

(2) Division of General Internal Medicine, Medical Effectiveness Research Center for Diverse Populations, Department of Medicine, University of California San Francisco (UCSF). Estados Unidos de América.

Fecha de recibido: 10 de septiembre de 2007 - Fecha de aceptado: II de abril de 2008 Solicitud de sobretiros: Dra. Ethel Alderete. Consejo Nacional de Investigaciones Científicas y Técnicas (CONICET). Lavalle 33 S.S. de Jury,Argentina. 4600 Argentina.

Correo electrónico:e_alderete@arnet.com.ar 


\section{Consecuencias sobre la salud}

El consumo excesivo de alcohol produce daños físicos, mentales y sociales y constituye una de las causas principales del cáncer de boca, esófago y laringe ${ }^{1-4}$ además de otros problemas médicos, entre ellos hipertensión, gastritis, cirrosis hepática y pancreatitis. El alcohol puede causar daños durante la gestación y agravar algunas formas del infarto cerebral y trastornos mentales, como la depresión. Asimismo, las lesiones por accidentes de tráfico y peatonales, las caídas, la violencia y los problemas laborales son consecuencia frecuente del consumo excesivo. ${ }^{5,6}$ Este último ocasiona enfermedad y sufrimiento no sólo en el bebedor sino también en su familia y allegados. ${ }^{7-10}$

La relación entre el consumo de alcohol y los daños a la salud dependen del volumen y la frecuencia de la ingestión y otros mecanismos más. Cuando se consumen en forma moderada, las bebidas alcohólicas pueden ser beneficiosas dado que atenúan la formación de coágulos sanguíneos, ${ }^{11}$ lo cual representa un mecanismo protector para las enfermedades coronarias. Por otro lado, la toxicidad por consumo excesivo puede producir daño pancreático ${ }^{12}$ y es un mediador importante de efectos agudos, como accidentes, lesiones intencionales o muerte y violencia familiar,; ${ }^{13,14}$ por su parte, la dependencia de alcohol es un mecanismo que influye sobre el consumo sostenido y se refleja en problemas crónicos y agudos. ${ }^{15}$ En suma, la cantidad ingerida es un factor relacionado sobre todo con las consecuencias a largo plazo, mientras que los patrones de consumo son mejores predictores de los efectos agudos. ${ }^{16-18}$ Por lo regular, el consumo excesivo comienza alrededor de los 13 años de edad y se observa un pico entre los $18 \mathrm{y}$ 22 años, seguido de un descenso gradual. ${ }^{19,20}$

\section{El alcoholismo y los jóvenes}

En Argentina, hasta la década 1980, el patrón cultural del consumo de alcohol era permisivo pero con rechazo a los excesos. A partir del año 2000, la permisividad incondicional se ha extendido en la sociedad. Este cambio es atribuible a la industria, en particular la productora de cerveza que multiplicó en grado notable sus ventas tras enfocar su publicidad en la población juvenil para ampliar el número de sus consumidores. El hábito de beber entre los jóvenes dejó de vincularse con la alimentación y el ambiente familiar y pasó a ser parte del encuentro nocturno, los lugares bailables, la diversión y el descontrol. ${ }^{21}$

En el año 2005, en una encuesta de prevalencia entre jóvenes argentinos de 14 a 17 años edad se determinó que $63 \%$ de los varones y 59\% de las mujeres habían consumido bebidas alcohólicas alguna vez en su vida y hasta 42 y $37 \%$, respectivamente, en el último mes mientras que $31 \%$ de los varones y $23 \%$ de las mujeres consumían en forma excesiva. Para la provincia de Jujuy, los resultados indicaban una prevalencia de consumo algo más baja. Hasta 48\% de los varones y 39\% de las mujeres habían consumido alguna vez en la vida, en tanto que $27 \%$ de los varones y $19 \%$ de las mujeres lo habían hecho en el último mes. ${ }^{22}$

Este estudio amplía los conocimientos existentes y describe, además de los patrones de consumo, los problemas relacionados con el consumo y los factores sociodemográficos vinculados con él en jóvenes de una población socialmente vulnerable.

\section{Material y métodos}

El estudio se llevó a cabo en la provincia de Jujuy al noroeste de Argentina, una zona fronteriza ubicada en la franja andino-amazónica, que acusa problemas de marginación y vulnerabilidad social. Una proporción de $44 \%$ de la población vive bajo la línea de pobreza y el desempleo alcanza una cifra de 25 por ciento. ${ }^{23} \mathrm{Si}$ bien la mayoría de la población es de ascendencia indígena, ${ }^{24,25}$ algunos se definen como mestizos, mezcla de indígenas y europeos; sólo una minoría tiene orígenes europeos.

En el año 2004 se aplicó la encuesta de base de un estudio longitudinal sobre tabaquismo en jóvenes que cursaban el octavo grado escolar. Se estratificó la muestra por zona geográfica (cordillera, valles, ciudad capital) debido a la existencia de variaciones étnicas y sociodemográficas. Se seleccionó al azar, de la lista que proporcionó el Ministerio de Educación, una muestra representativa de 27 escuelas con un total de 1000 alumnos por cada zona geográfica. En la encuesta de seguimiento del año 2005 (noveno grado) se incluyeron preguntas sobre consumo de alcohol. Un comité de ética certificado por los Institutos Nacionales de la Salud (NIH de Estados Unidos de América) aprobó el protocolo de investigación. De igual modo, se obtuvo consentimiento informado de padres y alumnos.

Para el desarrollo del cuestionario se utilizaron preguntas estandardizadas tomadas de cuestionarios existentes, como el empleado en el Sistema de Vigilancia de Comportamientos de Riesgo en Jóvenes (YRBSS) ${ }^{26}$ y el Instrumento de Evaluación para los Trastornos Debidos al Consumo de Alcohol (AUDIT), ${ }^{27}$ así como preguntas desarrolladas por el equipo y basadas en investigaciones cualitativas previas. Se llevaron a cabo evaluaciones de comprensión y pruebas piloto del instrumento. 


\section{Variables de efecto}

Se recabó información sobre cantidad y frecuencia de consumo en el último mes y última semana, edad de inicio, tipo de bebida y problemas relacionados con el consumo en la vida, como faltar a la escuela, consumir en las escuelas, padecer problemas con la familia, incurrir en altercados con la policía y sufrir lesiones.

El AUDIT es una prueba efectiva para detectar abuso o dependencia de alcohol en adultos ${ }^{28,29}$ y jóvenes. ${ }^{30}$ Para la identificación del consumo de riesgo, síntomas de dependencia y consumo perjudicial se aplicaron los algoritmos derivados de la versión abreviada (AUDITC), ${ }^{27}$ que evalúa el dominio consumo de riesgo mediante los factores frecuencia de consumo, cantidad habitual de consumo y frecuencia de consumo elevado durante el último mes ( $\geq 5$ medidas por ocasión). El dominio sintomas de dependencia se evaluó con los factores pérdida de control sobre el consumo e incremento de la importancia atribuida al consumo en el último año. El dominio consumo perjudicial se evaluó con el factor preocupación de otros por el consumo en la vida. Las preguntas aplicadas poseen respuestas con una escala de puntuación de 0 a 4 . Los algoritmos de las tres preguntas sobre consumo de riesgo se computan en una escala de 0 a 12 puntos. Para los varones, un puntaje de 4 o más se considera positivo, mientras que para las mujeres se considera positivo uno de 3 . Una puntuación mayor de 0 para las preguntas de los dominios síntomas de dependencia o consumo perjudicial indica la presencia de daños relacionados con el alcohol, o bien la presencia o inicio de dependencia de alcohol, respectivamente.

\section{Variables de exposición}

Se recabó información sobre sexo, edad, autoidentificación étnica (indígena, mezcla de indígena y europeo, o ascendencia europea), religión (católica, evangélica, otra), realización de trabajos pagados y repetición de grado. Los jóvenes informaron sobre el nivel de educación y condición laboral de sus padres o tutores (empleado, desocupado, receptor de asistencia pública, jubilado o pensionado). Se registró también información sobre el tipo de escuela (pública o privada), la orientación académica de la escuela (bachiller, magisterio, comercial, técnica, artística, agrícola), el turno escolar (mañana, tarde, noche) y la ubicación de la escuela (ciudades, $\geq 10000$ habitantes; pueblos, $<10000$ habitantes; o zonas rurales, habitantes dispersos).

\section{Análisis de datos}

Para el análisis de datos se empleó el programa computarizado SAS. * El diseño de la muestra se incorporó en el análisis, con especificación del área geográfica por estratos y escuelas como conglomerados. Se calculó la distribución de porcentajes y los errores estándar de variables demográficas para varones y mujeres y el total de la muestra. Se calculó la ji cuadrada y el valor $p$ para tablas de contingencia de las variables: consumo en la vida y el último mes, inicio de consumo a los 10 años o menor edad, consumo de riesgo en el último mes, presencia o aparición de dependencia de alcohol en el último año y consumo perjudicial en la vida. Se construyeron tres modelos de regresión logística con las variables consumo de riesgo, presencia o aparición de dependencia de alcohol y consumo perjudicial como variables de efecto, respectivamente. En los modelos se incluyeron variables sociodemográficas de los jóvenes, nivel de educación y situación laboral de los padres o tutores y características de la escuela.

\section{Resultados}

En las 27 escuelas seleccionadas en la encuesta de base se encontraban registrados 4284 alumnos del octavo grado. El porcentaje de respuesta para la encuesta fue de $86.1 \%$ con un total de 3690 alumnos; de éstos, 3466 $(80.9 \%)$ respondieron a la encuesta de seguimiento en noveno grado. Para este estudio se eliminó a 240 jóvenes mayores de edad (>17 años), 27 que se identificaron como árabes y 275 que no respondieron a la pregunta sobre autoidentificación étnica; el resultado fue una muestra de 2924 individuos.

\section{Características de la muestra}

Una proporción de $69.9 \%$ se autoidentificó como indígena y $22.5 \%$ como mezcla de indígena y europeo, en tanto que $7.6 \%$ lo hizo como europeo. Hasta 23\% había repetido el grado, $44 \%$ de los padres o tutores tenía nivel educativo primario o no había alcanzado ese nivel y $21 \%$ correspondía a desocupados o receptores de asistencia pública (cuadro I).

\footnotetext{
* SAS. SAS Institute Inc. Cary, NC, USA: SAS Institute Inc., 2006.
} 
Cuadro I

Características sociodemográficas de los jóvenes escolarizados de 12 a 17 años. Provincia de Jujuy, Argentina 2005

\begin{tabular}{|c|c|c|c|c|c|c|}
\hline & $\begin{array}{c}\text { Muj } \\
N=I \\
(53 . \\
\% \text { (s }\end{array}$ & $\begin{array}{l}\text { jeres } \\
562 \\
.3 \%) \\
\text { s.e.) }\end{array}$ & $\begin{array}{c}\text { Varo } \\
N=1 \\
(46.7 \\
\% \text { s. }\end{array}$ & $\begin{array}{l}\text { ones } \\
1362 \\
.7 \%) \\
\text { s.e.) }\end{array}$ & & $\begin{array}{l}\text { Total } \\
=2924 \\
\% \text { (s.e.) }\end{array}$ \\
\hline \multicolumn{7}{|l|}{ Características de los jóvenes } \\
\hline \multicolumn{7}{|l|}{ Edad } \\
\hline $12-14$ años & 62.8 & (3.4) & 61.3 & (3.7) & 62.1 & I (3.4) \\
\hline I5-I7 años & 37.2 & (3.3) & 38.7 & $(3.7)$ & 37.9 & $9(3.4)$ \\
\hline \multicolumn{7}{|l|}{ Autoidentificación étnica } \\
\hline Europeo & 5.6 & $(1.9)$ & 9.9 & $(3.2)$ & 7.6 & $6(2.5)^{*}$ \\
\hline Mezcla de indígena y europeo & 21.9 & (3.4) & 23.2 & $(2.6)$ & 22.5 & $5(2.8)$ \\
\hline Indígena & 72.5 & $(4.4)$ & 66.8 & $(5.2)$ & 69.9 & $9(4.6)$ \\
\hline \multicolumn{7}{|l|}{ Religión } \\
\hline Católica & 85.1 & (I.4) & 85.8 & $(1.7)$ & 85.4 & $4(1.4)$ \\
\hline Evangélica & 11 & $(\mathrm{I} . \mathrm{I})$ & 10.1 & $(1.3)$ & 10.6 & $6(\mathrm{I} . \mathrm{I})$ \\
\hline Otra & 3.9 & $(0.5)$ & 4.1 & $(0.6)$ & 4 & $(0.5)$ \\
\hline Trabajó alguna vez en su vida & 28.6 & (3.9) & 46 & (3.9) & 36.7 & $7(3.7)^{*}$ \\
\hline Repitió algún grado en la escuela & 22.1 & $(2.3)$ & 23 & $(3.2)$ & 22.5 & $5(2.6)$ \\
\hline \multicolumn{7}{|l|}{ Características de padres o tutores } \\
\hline \multicolumn{7}{|l|}{ Educación } \\
\hline Primaria o menor & 47.4 & $(3.8)$ & 39.9 & $(5.1)$ & 44 & $(4.4)^{*}$ \\
\hline Secundaria & 34 & $(1.9)$ & 34.2 & $(3.1)$ & 34.1 & $(2.3)$ \\
\hline Técnica o universitaria & 18.5 & (4) & 25.8 & $(6.6)$ & 21.9 & $9(5.3)$ \\
\hline \multicolumn{7}{|l|}{ Situación laboral } \\
\hline Empleado & 68.7 & $(2.6)$ & 74 & $(2.3)$ & 71.1 & $(2.5)^{*}$ \\
\hline Desempleado & 18.5 & $(1.6)$ & 13.3 & $(1.8)$ & 16.1 & $(1.7)$ \\
\hline Recibe asistencia pública & 5.2 & $(0.5)$ & 5.4 & $(0.8)$ & 5.3 & $3(0.6)$ \\
\hline Jubilado & 7.6 & $(1.2)$ & 7.3 & (I) & 7.4 & $4(1.1)$ \\
\hline \multicolumn{7}{|l|}{ Características de la escuela } \\
\hline \multicolumn{7}{|l|}{ Tipo de escuela } \\
\hline Pública & 90 & $(5.9)$ & 83.1 & $(9.8)$ & 86.8 & $8(7.8)$ \\
\hline Privada & 10 & (5.9) & 17 & $(9.8)$ & 13.2 & $2(7.8)$ \\
\hline \multicolumn{7}{|l|}{ Tipo de enseñanza } \\
\hline Bachillerato & 46 & (13.2) & $45.1(1$ & (I2.4) & 45.6 & $6(12.6)$ \\
\hline Magisterio & 4.6 & $(1.7)$ & 4.6 & $(1.7)$ & 4.6 & $6(1.7)$ \\
\hline Comercial & 40.5 & $(14.1)$ & $36.2(1$ & $(13.0)$ & 38.5 & $5(13.5)$ \\
\hline Técnica & 5.7 & $(4.7)$ & 10 & $(7.2)$ & 7.7 & $7(5.9)$ \\
\hline Artística & 2.3 & (2) & 3 & $(2.5)$ & 2.6 & $6(2.2)$ \\
\hline Agrícola & 0.8 & $(0.8)$ & 1.1 & (I) & I & $(0.9)$ \\
\hline \multicolumn{7}{|l|}{ Turno } \\
\hline Mañana & 55.9 & $(5.4)$ & 58.4 & $(6.6)$ & 57 & $(5.8)$ \\
\hline Tarde & 33.5 & $(4.2)$ & 28.5 & $(4.4)$ & 31.2 & $2(4.2)$ \\
\hline Noche & 10.6 & $(4.1)$ & 13.1 & $(5.3)$ & 11.8 & $3(4.6)$ \\
\hline \multicolumn{7}{|l|}{ Ubicación geográfica } \\
\hline Urbana & 65 & $(8.7)$ & 69.1 & $(6.8)$ & 66.9 & $(7.8)$ \\
\hline Pueblo & 26.1 & $(9.9)$ & 20.2 & $(7.4)$ & 23.4 & $4(8.8)$ \\
\hline Rural & 8.9 & (3.6) & 10.6 & (3.9) & 9.7 & $7(3.7)$ \\
\hline
\end{tabular}

*i cuadrada; $p \leq 0.05$

\section{Consumo de bebidas alcohólicas}

No se encontraron diferencias significativas en la proporción de mujeres y varones que probaron bebidas alcohólicas alguna vez en la vida (53-55\%), que bebieron en los últimos 30 días $(24-27 \%)$ o que iniciaron el consumo a edad temprana ( $\leq 10$ años) (79\%) (cuadro II). Los jóvenes de mayor edad, religión católica, repetidores de grado, trabajadores e hijos de padres con empleo notificaron en mayor proporción el consumo en la vida o el último mes. Por el contrario, los jóvenes de menor edad, los que no habían repetido grado, los hijos de padres con educación técnica o universitaria y los asistentes al turno escolar matutino refirieron en mayor proporción el inicio del consumo a edad temprana. La bebida preferida fue la cerveza en $33 \%$ de los jóvenes, seguida por el vino $(13 \%)$ y las bebidas de mayor contenido alcohólico (fernet, vodka, whisky) (13\%).

\section{Problemas relacionados con el consumo}

Los problemas con la familia $(7.1 \%)$ y las lesiones (5.5\%) fueron los más comunes, seguidos por el ausentismo escolar $(5.1 \%)$, consumo de bebidas en la escuela (3.8\%) o los problemas con la policía (3.4\%). Una proporción de 15\% de los jóvenes señaló que había sufrido alguno de estos problemas en la vida y los más afectados fueron los varones (18\%). Comparados con los hijos de padres desempleados, los jóvenes con padres empleados informaron en mayor proporción consumo de riesgo (10.1 contra $4.6 \%$ ) y los hijos de padres receptores de asistencia pública refirieron en mayor proporción síntomas de dependencia (17.2 contra 11.4\%). Un mayor porcentaje de varones, jóvenes que trabajaban y repetidores de grado registraron síntomas de dependencia y consumo perjudicial; en cambio, tener mayor edad y asistir al turno escolar nocturno resultaron variables vinculadas con el consumo de riesgo, síntomas de dependencia y consumo perjudicial (cuadro II).

En modelos de regresión logística, la razón de probabilidades para las mujeres respecto de tener una puntuación positiva para los síntomas de dependencia (RM ajustada 0.7; IC95\% 0.6-0.8) y consumo perjudicial ( $R M$ ajustada 0.7; IC95\% 0.6-0.8) fue menor en grado significativo en comparación con los varones (cuadro III). Sin embargo, no hubo diferencia relevante por sexo para el consumo de riesgo. Tener mayor edad, trabajar y asistir a escuelas de turno nocturno fueron los factores vinculados con las tres variables de efecto. Los jóvenes que asistían a escuelas de zonas rurales presentaban 


\section{Cuadro II}

\section{CONSUMO DE BEBIDAS ALCOHÓLICAS POR CARACTERÍSTICAS SOCIODEMOGRÁFICAS EN JÓVENES ESCOLARIZADOS de 12 a 17 años. Provincia de Jujuy,Argentina 2005}

\begin{tabular}{|c|c|c|c|c|c|c|}
\hline \multirow[t]{3}{*}{ 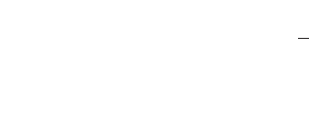 } & \multicolumn{3}{|c|}{ Patrones de consumo } & \multicolumn{3}{|c|}{ Problemas relacionados con el consumo } \\
\hline & $\begin{array}{l}\text { Consumo en la vida } \\
N=I 525 \text { (53.7\%) }\end{array}$ & $\begin{array}{l}\text { Consumo en el último mes } \\
\qquad N=7 / 8(25.3 \%)\end{array}$ & $\begin{array}{c}\text { Edad de inicio ( } \leq 10 \text { años) } \\
\quad N=119(7.8 \%)\end{array}$ & $\begin{array}{c}\text { Consumo de riesgo } \\
N=290 \text { (9.9\%) }\end{array}$ & $\begin{array}{l}\text { Sintomas de dependencia } \\
\quad N=447(15.2 \%)\end{array}$ & $\begin{array}{r}\text { Consumo perjudicial } \\
N=415 \text { (I3.8\%) }\end{array}$ \\
\hline & $\%$ (s.e.) & $\%$ (s.e.) & \% (s.e.) & \% (s.e.) & \% (s.e.) & $\%$ (s.e.) \\
\hline \multicolumn{7}{|l|}{ Características de los jóvenes } \\
\hline \multicolumn{7}{|l|}{ Sexo } \\
\hline Mujeres & $53 \quad(1.6)$ & $23.5(1.7)$ & $6.9(1.1)$ & $9.3(0.9)$ & $12.3(\mathrm{I} .1)^{*}$ & $10.9(0.8)^{*}$ \\
\hline Varones & $54.7(3.2)$ & $27.3(3.3)$ & $8.7(1.3)$ & $10.6(I)$ & $18.5(1.2)$ & $17.2(1.8)$ \\
\hline \multicolumn{7}{|l|}{ Edad } \\
\hline $12-14$ & $49.3(2.6)^{*}$ & $21.6(2.3)^{*}$ & $9.9(\mathrm{I})^{*}$ & $7.5(0.7)^{*}$ & $11.3(\mathrm{I}) *$ & $10.4(\mathrm{I}) *$ \\
\hline $15-17$ & $61.1(1.8)$ & $31.4(1.4)$ & $5 \quad(I)$ & $13.8(0.9)$ & $21.4(1.4)$ & $19.4(1.2)$ \\
\hline \multicolumn{7}{|l|}{ Autoidentificación étnica } \\
\hline Europeo & $65.1(5.3)$ & $34.3(4.5)$ & $8.5(1.4)$ & $12.6(1.6)$ & I3.6 (I.9) & $7.5(1.9)$ \\
\hline Indígena y europeo & $57.5(3.3)$ & $28.4(2.6)$ & $7.2(1.4)$ & II.3(0.9) & $15.4(1.3)$ & $14.8(1.4)$ \\
\hline Indígena & $51.3(1.4)$ & $23.3(1.4)$ & $7.9(\mathrm{I})$ & $9.2(0.9)$ & $14.9(1.4)$ & $12.8(1.5)$ \\
\hline \multicolumn{7}{|l|}{ Religión } \\
\hline Católica & $55 \quad(2.3)^{*}$ & $26.2(2)^{*}$ & $8.1(1.0)$ & $9.9(0.8)$ & $15.6(1.2)$ & I4.I (I.3) \\
\hline Evangélica & $42.9(2.6)$ & $20.9(2.5)$ & $4.6(1.4)$ & $10.8(2)$ & $13 \quad(1.9)$ & $13.3(2.1)$ \\
\hline Otra & $54.2(3.3)$ & $16.6(3.2)$ & $6.8(2.5)$ & $8.0(2.3)$ & $10.8(2.8)$ & $7.9(2.9)$ \\
\hline \multicolumn{7}{|l|}{ Trabajó alguna vez en su vida } \\
\hline Sí & $61.2(1.8)^{*}$ & $31.1 \quad(1.2)^{*}$ & $6.9(1.1)$ & I3.8 (I)* & $21.7(1.6)^{*}$ & $21.1 \quad(1.7)^{*}$ \\
\hline No & $50.1(3.1)$ & $22.2(2.6)$ & $8.3(1.2)$ & $7.8(0.6)$ & $11.6(0.6)$ & $9.7(0.6)$ \\
\hline \multicolumn{7}{|l|}{ Repitió un grado en la escuela } \\
\hline Sí & $62.3(1.9)^{*}$ & $32 \quad(1.6)^{*}$ & $4 \quad(0.9)^{*}$ & $14 \quad(1.2)^{*}$ & $23.2(2.2)^{*}$ & $21.7(1.6)^{*}$ \\
\hline No & $51 \quad(2.7)$ & $23.3(2.5)$ & $9.2(\mathrm{I})$ & $8.6(0.8)$ & $12.6(0.9)$ & $\mathrm{II} .4(\mathrm{I} .0)$ \\
\hline \multicolumn{7}{|c|}{ Características de padres o tutores } \\
\hline \multicolumn{7}{|l|}{ Educación } \\
\hline Primaria o menor & $52.5(1.6)$ & $24.1(I)$ & $6.7(0.8)^{*}$ & $9.7(0.9)$ & $16.5(I)$ & $15.2(0.9)$ \\
\hline Secundaria & $5 \mathrm{I.I}(\mathrm{I} .7)$ & $25.3(1.6)$ & $6.1(1.1)$ & $10.3(I . I)$ & $14.9(1.3)$ & $14.2(1.8)$ \\
\hline Técnica o universitaria & $61.8(5.0)$ & $28.4(4.5)$ & II.6 (I.5) & $9.9(1.5)$ & I3.I (I.5) & $10.3(1.4)$ \\
\hline \multicolumn{7}{|l|}{ Situación laboral } \\
\hline Empleado & $54.5(2.4)^{*}$ & $26.5(2.1)^{*}$ & $8.6(1.3)$ & $10.1(0.8)^{*}$ & $14.7(1.2)^{*}$ & I3.3 (I.2) \\
\hline Desempleado & $46.8(4.8)$ & $17.7(3)$ & $6.1(3)$ & $4.6(1.3)$ & II.4 (2.5) & $13.4(3.0)$ \\
\hline Recibe asistencia pública & $49.6(2.2)$ & $21.8(1.4)$ & $4.9(1.1)$ & $9.7(1.4)$ & $17.2(\mathrm{I})$ & $14.5(1.5)$ \\
\hline Jubilado & $62.7(4.6)$ & $28.2(3.3)$ & $5.3(1.7)$ & $10.7(1.6)$ & $19 \quad(2.1)$ & $18.4(2.1)$ \\
\hline \multicolumn{7}{|l|}{ Características de las escuelas } \\
\hline \multicolumn{7}{|l|}{ Tipo de escuela } \\
\hline Pública & $51.4(1.2)$ & $23.3(1.2)$ & $6.7(0.8)$ & $9.7(0.8)$ & $15.6(1.2)$ & $14.6(1.3)$ \\
\hline Privada & $69 \quad(5.4)$ & $38.4(3.4)$ & $12.9(0.8)$ & II.4 (0.7) & $12.3(1.6)$ & $8.8(1.3)$ \\
\hline \multicolumn{7}{|l|}{ Tipo de instrucción } \\
\hline Bachillerato & $58.4(3.8)$ & $29.7(3.1)$ & $8.1(1.6)$ & $10.8(0.8)$ & I5.| (I.4) & $11.2(1.2)$ \\
\hline Magisterio & $42.3(2.2)$ & $19.2(\mathrm{I})$ & $7.0(0.5)$ & $9.2(0.6)$ & $16.2(1.1)$ & $16.9(1.2)$ \\
\hline Comercial & $50.7(1.4)$ & $21.7(2.1)$ & $7.8(1.3)$ & $8.8(1.6)$ & I4.I (I.9) & $15.8(1.7)$ \\
\hline Técnica & $54.8(1.4)$ & $21.1(0.3)$ & $6.5(0.7)$ & $10.2(0.9)$ & $17.3(\mathrm{I} . \mathrm{I})$ & $14.8(I)$ \\
\hline Artística & $28 \quad(2.5)$ & $12 \quad(1.7)$ & $9 \quad(1.5)$ & $7.2(0.8)$ & $9.7(1.9)$ & $10.5(1.2)$ \\
\hline Agrícola & $63.3(0)$ & $43.3(0)$ & $10.5(0)$ & $26.7(0)$ & $23.3(0)$ & $16.7(0)$ \\
\hline \multicolumn{7}{|l|}{ Turno } \\
\hline Mañana & $53.8(3.5)^{*}$ & $24.3(3.0)$ & $9.8(I . I)^{*}$ & I8.I (I.4)* & $12.4(1.1)^{*}$ & II.4 (1.2)* \\
\hline Tarde & $47.9(1.9)$ & $22.6(2.1)$ & $6.6(1.9)$ & $21.8(2.6)$ & $14.6(2.0)$ & I4.I (I.8) \\
\hline Noche & $61.1(3.8)$ & $31.3(4.8)$ & $4.5(1.5)$ & $36.0(4.9)$ & $26.5(3.2)$ & $21.5(3.5)$ \\
\hline \multicolumn{7}{|l|}{ Ubicación geográfica } \\
\hline Urbana & $54.2(3.1)$ & $24.4(2.7)$ & $8.0(1.2)$ & $21.2(1.9)$ & 14.7 (I.4) & $12.8(1.4)$ \\
\hline Pueblo & $50.5(1.3)$ & $26.2(1.2)$ & $7.4(1.8)$ & 22.7 (I.5) & $15.1(1.2)$ & $15.6(1.2)$ \\
\hline Rural & $58.8(4.7)$ & 29.1 (2.6) & $6.7(0.8)$ & $24.1 \quad(2.4)$ & $18.3(2.2)$ & $16.3(3.1)$ \\
\hline
\end{tabular}




\section{Cuadro III}

Modelos multivariados de Regresión logística para consumo de bebidas alcohólicas en jóvenes escolarizados de 12 a 17 años. Provincia de Jujuy,Argentina 2005

\begin{tabular}{|c|c|c|c|}
\hline & $\begin{array}{c}\text { Consumo de riesgo } \\
\text { RM (IC95\%) }\end{array}$ & $\begin{array}{c}\text { Sintomas de dependencia } \\
\text { RM (IC95\%) }\end{array}$ & $\begin{array}{l}\text { Consumo perjudicial } \\
\text { RM (IC95\%) }\end{array}$ \\
\hline \multicolumn{4}{|l|}{ Características de los jóvenes } \\
\hline \multicolumn{4}{|l|}{ Sexo } \\
\hline Mujeres o varones & I.I $(0.9-1.4)$ & $0.7(0.6-0.8)^{*}$ & $0.7(0.6-0.8)^{*}$ \\
\hline \multicolumn{4}{|l|}{ Edad en años } \\
\hline $12-14 \circ 15-17$ & $0.6(0.5-0.7)^{*}$ & $0.7(0.6-0.9)^{*}$ & $0.7(0.5-0.9)^{*}$ \\
\hline \multicolumn{4}{|l|}{ Autoidentificación étnica } \\
\hline Europeo o mezcla & $1.3(0.8-2.1)$ & I.I (0.7-I.7) & $0.6(0.4-I)$ \\
\hline Indígena o mezcla & $0.9(0.7-1.3)$ & I.I (0.8-I.4) & $1.2(0.9-1.6)$ \\
\hline \multicolumn{4}{|l|}{ Religión } \\
\hline Católica u otra & I.I $(0.5-2.4)$ & $1.7(0.9-3.3)$ & $1.7(0.7-4.1)$ \\
\hline Evangélica u otra & $1.2(0.5-2.9)$ & $1.2(0.6-2.5)$ & $1.2(0.5-3.2)$ \\
\hline \multicolumn{4}{|l|}{ Situación laboral } \\
\hline Trabajó alguna vez en su vida o no trabajó & $1.6(1.3-2.0)^{*}$ & $1.6(1.3-2.1)^{*}$ & $1.8(1.5-2.3)^{*}$ \\
\hline \multicolumn{4}{|l|}{ Repitió un grado en la escuela } \\
\hline No o Sí & I (0.8-1.3) & I. $(0.8-1.2)$ & $0.8(0.7-1 . I)$ \\
\hline \multicolumn{4}{|l|}{ Características de padres o tutores } \\
\hline \multicolumn{4}{|l|}{ Educación } \\
\hline Primaria o menos contra técnica o universitaria & $0.9(0.6-1.3)$ & I.I $(0.7-1.5)$ & I.I $(0.7-1.6)$ \\
\hline Secundaria contra técnica o universitaria & I $\quad(0.7-1.4)$ & I $\quad(0.7-1.4)$ & $1.2(0.7-1.8)$ \\
\hline \multicolumn{4}{|l|}{ Situación laboral } \\
\hline Empleado o jubilado & I $\quad(0.6-1.6)$ & $0.9(0.7-1.1)$ & $0.8(0.6-1.2)$ \\
\hline Recibe asistencia pública o jubilado & I $(0.6-1.7)$ & $0.9(0.7-1.3)$ & $0.8(0.5-1.3)$ \\
\hline Desempleado o jubilado & $0.4(0.2-I)$ & $0.6(0.3-1)$ & $0.7(0.4-1.3)$ \\
\hline \multicolumn{4}{|l|}{ Características de la escuela } \\
\hline \multicolumn{4}{|l|}{ Tipo de escuela } \\
\hline Pública o privada & $1.4(0.9-2)$ & $0.9(0.6-1.5)$ & I.I $(0.6-2.2)$ \\
\hline \multicolumn{4}{|l|}{ Tipo de instrucción } \\
\hline Bachiller o agrícola & $0.9(0.7-1.2)$ & $0.5(0.3-0.7)^{*}$ & $0.6(0.4-0.8)^{*}$ \\
\hline Magisterio o agrícola & I $(0.6-I .4)$ & $0.7(0.4-I . I)$ & I $\quad(0.6-1.6)$ \\
\hline Comercial o agrícola & $0.7(0.5-1)$ & $0.5(0.3-0.7)^{*}$ & $0.8(0.5-1.2)$ \\
\hline Técnica o agrícola & $0.7(0.5-1.2)$ & $0.3(0.2-0.5)^{*}$ & $0.4(0.3-0.7)^{*}$ \\
\hline Artística o agrícola & $0.7(0.5-I . I)$ & $0.3(0.2-0.4)^{*}$ & $0.5(0.3-0.7)^{*}$ \\
\hline \multicolumn{4}{|l|}{ Turno } \\
\hline Mañana o noche & $0.5(0.4-0.7)^{*}$ & $0.4(0.3-0.6)^{*}$ & $0.6(0.4-0.8)^{*}$ \\
\hline Tarde o noche & $0.7(0.5-0.9)^{*}$ & $0.4(0.3-0.6)^{*}$ & $0.6(0.4-0.9)^{*}$ \\
\hline \multicolumn{4}{|l|}{ Ubicación geográfica } \\
\hline Urbana o rural & $0.8(0.6-1.1)$ & $0.9(0.6-1.3)$ & $0.8(0.6-1.3)$ \\
\hline Pueblo o rural & $0.7(0.5-I)$ & $0.6(0.4-0.9)^{*}$ & $0.7(0.5-\mathrm{I} . \mathrm{I})$ \\
\hline
\end{tabular}

mayor riesgo de mostrar síntomas de dependencia respecto de los que asistían a escuelas en pueblos. Asimismo, asistir a una escuela con orientación agrícola, en comparación con otro tipo de orientación escolar, demostró ser una variable significativa para síntomas de dependencia y consumo perjudicial.

\section{Discusión}

Los resultados que son representativos de la población que asiste al noveno grado indican que el consumo de bebidas alcohólicas en jóvenes de la provincia de Jujuy es algo menor que el consumo nacional y similar al 
informado para jóvenes de otras regiones de Latinoamérica. En el estado de Hidalgo, México, por ejemplo, el consumo en la vida fue de $48 \%$ y el consumo excesivo de 13 por ciento. ${ }^{31}$ Las características sociodemográficas de la presente muestra confirman su condición de población vulnerable. Futuros estudios deben analizar las características sociodemográficas, patrones culturales de consumo y contexto social que marcan similitudes y diferencias en relación con los comportamientos de riesgo en jóvenes de regiones marginales de un país respecto de sus áreas metropolitanas, así como las similitudes y diferencias entre regiones no metropolitanas de los distintos países de Latinoamérica.

En este estudio se identificó que algunos parámetros de consumo son más comunes entre los varones, por ejemplo, presentar síntomas de dependencia o consumo perjudicial. Sin embargo, para otros parámetros como el consumo de riesgo, no existen diferencias significativas entre varones y mujeres. El consumo excesivo en mayor proporción de varones que de mujeres se ha notificado en los protocolos de Rojas-Guiot y colaboradores ${ }^{31}$ y Natera-Rey y colaboradores ${ }^{32}$ en jóvenes mexicanos, mientras que en España Ariza y colaboradores ${ }^{33}$ informaron una prevalencia similar de problemas relacionados con el consumo entre mujeres y varones.

En concordancia con la bibliografía existente, el consumo de bebidas alcohólicas en este estudio aumenta con la edad. ${ }^{22,34} \mathrm{El}$ inicio del consumo a edad temprana es más común en la cohorte de 12 a 14 años de edad y entre jóvenes con un mejor nivel socioeconómico y desempeño escolar, pero al parecer en esta muestra no sería indicativo de consumo problemático.

Los resultados coinciden con los de las publicaciones disponibles, según las cuales una mayor proporción de jóvenes que trabajan padece un consumo problemático. ${ }^{35,36}$ Asimismo, investigaciones previas en la región indican que los jóvenes de los turnos escolares nocturnos tienen mayor riesgo de consumir sustancias psicotrópicas. ${ }^{37}$ Los resultados existentes que evalúan la relación entre los patrones de consumo y la condición socioeconómica no son concluyentes. En Chile, Nader y colaboradores ${ }^{38}$ comunicaron una mayor prevalencia de alcoholismo en jóvenes de baja condición socioeconómica, si bien en un estudio realizado en adolescentes escolarizados en una zona urbana del sureste de Brasil no se reconocieron diferencias significativas por clase social. ${ }^{39}$

No se hallaron diferencias de importancia en los patrones de consumo o problemas vinculados en relación con la autoidentificación étnica de la muestra. No se encontraron publicaciones que examinaran la relación entre el consumo de bebidas alcohólicas y la etnicidad en jóvenes de la región sudamericana. En una encuesta poblacional en adultos de Bahía, Brasil, no se identificaron diferencias significativas, aunque la demografía del área, con una contribución étnica considerable de descendientes africanos, es muy diferente a la de este estudio. ${ }^{40}$

Una limitación del presente protocolo es la concentración de las entrevistas en jóvenes escolarizados, que podrían presentar una menor prevalencia de consumo que los sujetos no escolarizados, quienes pueden estar expuestos a condiciones de vida que incrementen su proclividad a consumir sustancias psicotrópicas. Sin embargo, según datos del censo 2001, sólo $0.8 \%$ de los jóvenes de 10 a 14 años de edad en el área no asistía a la escuela. ${ }^{23}$ Además, no se realizó una evaluación diagnóstica de dependencia de alcohol.

Pese a todo, esta investigación ha determinado que una proporción notoria de los jóvenes consume bebidas alcohólicas desde temprana edad y en forma excesiva, presenta síntomas de dependencia y experimenta problemas relacionados con el consumo que inciden en su vida personal, familiar y escolar. Estos hallazgos ponen de manifiesto la necesidad de instituir medidas de prevención y tratamiento de alcoholismo, enfocados y adaptados a la población adolescente.

\section{Agradecimientos}

Este estudio recibió financiamiento del proyecto No. TW05935 del Tobacco Research Network Program, Fogarty International Center, National Cancer Institute, National Institute of Drug Abuse, National Institutes of Health, Estados Unidos de América y el proyecto No. 001726-037 del Research on International Tobacco Control, International Development Research Center, Canadá.

\section{Referencias}

I. English DR. The quantification of drug caused morbidity and mortality in Australia, 1992. Camberra: Commonwealth Department of Human Services and Health, 1995.

2. Gutjahr E, Gmel G, Rehm J. Relation between average alcohol consumption and disease: an overview. Eur Addict Res 200 I;7(3): I 17-1 27. 3. Ridolfo $B$, Stevenson CE. The quantification of drug-caused mortality and morbidity in Australia, 1998. Camberra:Australian Institute of Health and Welfare, 200I.

4. Single E, Robson L, Rehm J, Xie X. Morbidity and mortality attributable to alcohol, tobacco, and illicit drug use in Canada. Am J Public Health 1999;89(3):385-390.

5. Slater MD, Long M, Ford VL.Alcohol, illegal drugs, violent crime, and traffic-related and other unintended injuries in US local and national news. J Stud Alcohol 2006;67(6):904-910. 
6. Eaton DK, Kann L, Kinchen S, Ross J, Hawkins J, Harris WA, et al. Youth risk behavior surveillance-United States 2005.J Sch Health 2006;76(7):353-372.

7. Tempier R, Boyer R, Lambert J, Mosier K, Duncan CR. Psychological distress among female spouses of male at-risk drinkers. Alcohol 2006;40(I):4I-49.

8. World Health Organisation. Problems related to alcohol consumption. Report of a WHO Expert Committee. Tech. Report Series 650, Geneva: WHO, 1980.

9. Rehm J,Taylor B, Room R. Global burden of disease from alcohol, illicit drugs and tobacco. Drug Alcohol Rev 2006;25(6):503-5I3.

I0. Ezzati M, Lopez AD, Rodgers A,Vander-Hoorn S, Murray CJ.

Comparative Risk Assessment Collaborating Group. Selected major risk factors and global and regional burden of disease. Lancet 2002;360(9343):1347-1360.

II. Zakhari S.Alcohol and the cardiovascular system: molecular mechanisms for beneficial and harmful action. Alcohol Health Res World 1997;21:21-29.

12. Apte MV,Wilson JS, Korsten MA.Alcohol-related pancreatic damage: mechanisms and treatment. Alcohol Health Res World 1997;2I(I):I3-20.

13. Klingemann $\mathrm{H}, \mathrm{Gmel} \mathrm{G}$, eds. Mapping the social consequences of alcohol consumption. Dordrecht: Kluwer Academic Publishers, 200I. 14. Gmel G, Rehm J. Harmful alcohol use.Alcohol Res Health 2003;27(I):52-62.

15. Drummond DC. The relationship between alcohol dependence and alcohol-related problems in a clinical population. Brit J Addic 1990;85(3):357-366.

16. Rehm J,Ashley MJ, Room R, Single E, Bondy S, Ferrence R, et al. On the emerging paradigm of drinking patterns and their social and health consequences. Addiction 1996;91(II):1615-1621.

17. Bondy SJ. Overview of studies on drinking patterns and their reported consequences.Addiction 1996;9I(I I):1661-1674.

18. Puddey IB, Rakic V, Dimmitt SB, Beilin LJ. Influence of pattern of drinking on cardiovascular disease and cardiovascular risk factors - a review.Addiction 1999;94(5):649-663.

19. Johnston LD, O'Malley P, Bachman JG. National Survey Results on Drug Use From the Monitoring the Future Study, 1975-1994: Secondary School Students. Rockville, MD: National Institute on Drug Abuse, 1995;Vol.I. 20. Maggs JL, Schulenberg JE. Initiation and course of alcohol consumption among adolescent and young adults. Rec Dev Alcohol 2005; 17:29-47. 21. Kornblit AL, Mendes Diz AM, Di Leo PF, Camarotti AC,Adasko D. Salud y enfermedad desde la perspectiva de los jóvenes. Un estudio en jóvenes escolarizados de la Ciudad de Buenos Aires. Documento de Trabajo. Buenos Aires, Argentina: Instituto Gino Germani, 2005.

22. SEDRONAR Segunda Encuesta Nacional de estudiantes de enseñanza media 2005. Buenos Aires: Secretaría de Programación para la Prevención de la Drogadicción y la Lucha contra el Narcotráfico, 2006.

23. INDEC, Censo Nacional de Población, Hogares y Viviendas 2001. Buenos Aires: Instituto Nacional de Estadísticas y Censo, 200I.

24. Dugoujon S, Dipierri A, Ocampo J. Los andinos de Jujuy: estructura genética y niveles altitudinales. Lima, Perú: IX Congreso Latinoamericano de Genética, 1984:25.
25. Organización Internacional del Trabajo. Convenio 107. Disponible en: http://www.cesdepu.com/instint/OITI07.htm.

26. Eaton DK, Kann L, Kinchen S, Ross J, Hawkins J, Harris W. Youth Risk Behavior Surveillance-United States, 2005. Morb Mortal Wkly Report 2006;55(SS-5): I-108.

27. Bush K, Kivlahan DR, McDonell MB, Fihn SD, Bradley KA. The AUDIT alcohol consumption questions (AUDIT-C): an effective brief screening test for problem drinking. Arc Intern Med 1998;158:189-195.

28. Bradley KA, Bush KR, McDonell MB, Malone T, Fihn SD. The Ambulatory Care Quality Improvement Project (ACQUIP). Screening for problem drinking: comparison of CAGE and AUDIT.J Gen Intern Med 1998;13(6):39-388.

29. Bradley KA, Bush KR, Epler AJ, Dobie J, Davis TM, Sporleder JL, et al. Two brief alcohol screening tests from the Alcohol Use Disorders Identification Test (AUDIT): validation in a female VA patient population. Arch Intern Med 2003;163:821-839.

30. Chung T, Colby SM, Barnett NP, Rohsenow DJ, Spirito A, Monti PM. Screening adolescents for problem drinking: performance of brief screens against DSM-IV alcohol diagnoses. J Stud Alcohol 2000;6I (4):579-587.

31. Rojas-Guiot E, Fleiz-Bautista C, Medina-Mora Icaza ME, Morón MA,

Domenech-Rodríguez M. Consumo de alcohol y drogas en estudiantes de Pachuca, Hidalgo. Salud Publica Mex 1999;4I(4):297-308.

32. Natera-Rey GM, Borges G, Medina-Mora MEl, Solís-Rojas L,TiburcioSainz M. La influencia de la historia familiar de consumo de alcohol en hombres y mujeres. Salud Publica Mex 200I;43(I):17-26.

33. Ariza M, Nebot JR, Díez-Villalbí E,Tomás Z,Valmayor S. Tendencias en el consumo de tabaco, alcohol y cannabis de los escolares de Barcelona 1987-1999. Gac Sanit Barcelona 2003;17(3):190-195.

34. Pérula-de Torres LA, Ruiz-Moral R, Fernández-García JA, HerreraMorcillo E, de Miguel-Vázquez MD, Bueno-Cobo JM. Consumo de alcohol entre los escolares de una zona básica de salud de Córdoba. Rev Esp Salud Publica 1998;72(4):33I-334.

35. Urquieta JE, Hernández-Avila M, Hernández B. El consumo de tabaco y alcohol en jóvenes de zonas urbanas marginadas de México. Un análisis de decisiones relacionadas. Salud Publica Mex 2006;48(3):30-40.

36. Souza DP, Oliveira-de Areco KN, Silveira-Filho DX.Alcohol and alcoholism among brazilian adolescent public-school students. Rev Saude Publica 2005;39(4):585-592.

37. Soldera S, Meire-Dalgalarrondo P, Correa-Filho HR, Silva CA. Use of psychotropics drugs among students: prevalence and associated social factors. Rev Saude Publica Brasil 2004;38(2):277-283.

38. Nader A, Pallavicini G, Legarreta A, Mahaluf JZ, Cumsille FG, Silva CZ, et al. Epidemiología del hábito de beber en adolescentes. Rev Chil Pediatr 1985;56(4):217-22।.

39. Muza-Gilson M, Bettiol H, Muccillo G, Barbieri M. The intake of psychoactive substances by school-age adolescents in an urban area of southeastern region of Brazil: II. Distribution of consumption by social levels. Rev Saude Publica Brasil 1997;3I (2):163- 170.

40. Almeida-Filho N, Lessa I, Magalhaes L,Araújo MJ,Aquino E, Karachi I, et al.Alcohol drinking patterns by gender, ethnicity and social class in Bahia, Brazil. Rev Saude Publica Brasil 2004;38(I):45-54. 